\title{
Isaac syndrome; an unusual response to immunoglobulin therapy
}

\author{
Ethayakumar $\mathbf{N}^{1}$, Dissanayake $\mathrm{A}^{1}$, Pathirana $\mathrm{KD}^{2}$ \\ ${ }^{1}$ Teaching Hospital, Karapitiya, ${ }^{2}$ University Medical Unit, Karapitiya, Galle, Sri Lanka \\ Correspondence:Dr. N.Ethayakumar (drethayam@gmail.com)
}

\section{Introduction}

Isaac syndrome is characterised by spontaneous and continuous activity of muscle fibres. It is a very rare neurological condition and is due to the development of antibodies against the voltage-gated potassium channel (VGKC) of peripheral nerves (1). Most cases of Isaac syndrome occur sporadically. Patients with Isaac syndrome generally show a good therapeutic response to immunoglobulin therapy. Here we report a patient with Isaac syndrome, whose clinical features worsened with immunoglobulin therapy. We were unable to find a previous report of Isaac syndrome from Sri Lanka.

\section{Case Report}

A 29-year old male was admitted with increasing stiffness and numbness of muscles of both legs over the previous three months. The numbness and stiffness began in the feet, progressed to calves and thighs and later involved the arms and hands. It was associated with generalized body ache, muscle cramps especially in the legs, and profuse sweating. Chewing and swallowing were difficult occasionally and the chest had become stiff at times leading to some breathing difficulty. Stiffness of muscles was first induced by moderate effort and improved with rest, but condition had got progressively worsen and at the time of admission he was symptomatic even with minimal effort. He noticed twitching of thigh muscles while at rest to ameliorate stiffness. Duration of the muscle stiffness and twitching became prolonged gradually, and at time of admission they persisted for more than half an hour. He had no history of convulsions, hallucinations or double vision. His appetite and bowel habits were normal. He had a similar illness in his childhood for about eight months, and then remained well for the rest of his life. There was no family history of muscle disorders. He denied the use of alcohol and tobacco.
He was a well built man and there were visible involuntary twitching of muscles (myokymia), stiffness of both quadriceps induced by exertion and hyperhidrosis. During this episode there was a stocking type sensory impairment up to the mid calves. Power, tone, deep tendon reflexes and planter response were normal. Cranial nerves were normal and no cerebellar signs were elicited. Rest of the systemic examination was normal.

His serum calcium, electrolytes, thyroid function tests and CPK were within the normal limits. He had a normal full blood count. His fasting plasma glucose was $93 \mathrm{mg} / \mathrm{dL}$ while ESR was $6 \mathrm{~mm}$. His ALT and AST were 152 and $77 \mathrm{U} / \mathrm{L}$, respectively. Lipid profile was within the normal reference range. Abdominal ultrasonography showed fatty changes in the liver. His chest radiograph was normal. Nerve conduction studies showed polyphasic $\mathrm{F}$ waves, and electromyography showed doublets and triplets (Figure). With the characteristic clinical and electrophysiological findings, we arrived at a diagnosis of Isaac syndrome.

He was started on oral phenytoin sodium $100 \mathrm{mg}$ daily and was followed-up regularly. Numbness and hyperhidrosis improved a little but muscle cramps and twitching persisted after two months of treatment, hence he was given intravenous human immunoglobulin (IVIg) $0.4 \mathrm{~g} / \mathrm{kg} /$ day for five days. During IVIg therapy, he complained of severe muscle cramps, twitching and worsening of numbness, even with minimal effort. Therefore plasmapheresis was commenced and continued for two weeks and his symptoms improved. He was sent home with phenytoin sodium $200 \mathrm{mg}$ daily and was followed-up monthly. He became free of muscle cramps and twitching after three months but the numbness showed a lesser response. 


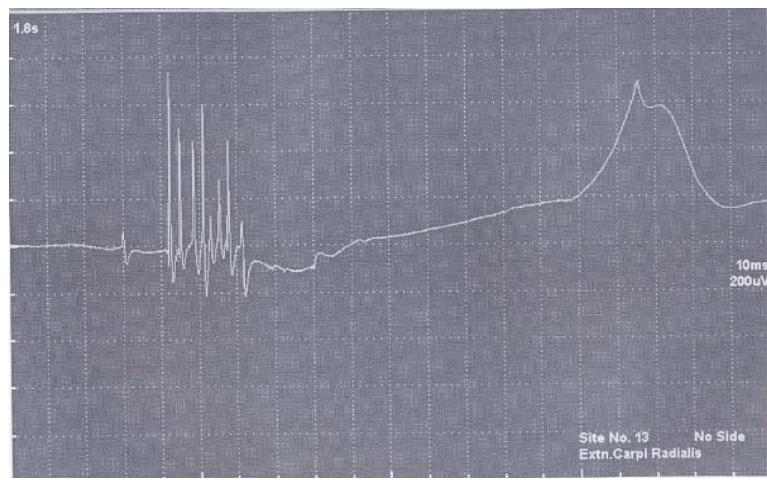

Figure: EMG showing the characteristic triplet / multiplet single motor unit discharges

\section{Discussion}

Isaac syndrome was first reported in 1961. The clinical hallmark of this syndrome is visible myokymia, which generally occurs in limbs but can occur in trunk muscles, face including tongue. Visible myokymia is seen in over $90 \%$ of patients and occasionally there is no visible muscle twitching at all, but needle electromyography reveals continuous motor unit activity (1).

Other clinical features of Isaac syndrome are muscle cramps, muscle stiffness, hyperhidrosis and pseudomyotonia, due to hyper-excitability of peripheral nerves including autonomic nerves (2), all of which were found in our patient. Deep tendon reflexes could be exaggerated or could be normal like in our patient. Confirmation of the syndrome needs neuro-electrophysiological studies where EMG reveals the characteristic doublet, triplet or multiplet single motor unit discharges (1) and the nerve conduction studies shows polyphasic $\mathrm{F}$ waves. Anti-VGKC antibodies are detected only in $40 \%$ of patients (1). There are few cases reported with the association of other autoimmune conditions and paraneoplastic syndrome.
Antiepileptic drugs those reduce peripheral nerve hyper-excitability, are the first choice of treatment but they usually give a temporary and partial improvement. Failure to achieve an adequate symptomatic control by antiepileptic drugs necessitates immunoglobulin therapy (3). Even though, in general, Isaac syndrome shows good results with immunoglobulin therapy, worsening of symptoms following immunoglobulin therapy has been reported (4). This is suspected to be due to direct effect of IVIg on muscles or hyper-excitability of muscles to IVIg (5). These cases have been treated with plasma exchange for up to four weeks (4). Incidentally, we noticed that both cases where symptoms got aggravated following immunoglobulin therapy had fatty changes in the liver.

\section{References}

1. Paul M. Neuromyotonia. Practical Neurol 2002; 2: 225-229.

2. Arimura K, Watanabe O. Immune mediated neuromytoniaclinical aspects and pathomechanism. Brain Nerve 2010; 62: 401-10.

3. Yuichi H, Akio K. Serial monitoring of BMR for therapeutic evaluation in an Isaacs' syndrome patient with chronic fluctuating symptoms. Inter Med 2010; 49: 475-7.

4. Ishii A, Hayashi A, Ohoshi N, et al. Clinical evaluation of plasma exchange and high dose intravenous immunoglobulin in a patient with Isaacs' syndrome. JNeurol Neurosurg Psychiatry 1994; 57: 840-2.

5. Van Engelen BG, Benders AA, Gabreels FJ, et al. Are muscle cramps in Isaacs' syndrome triggered by immunoglobulin? J Neurol Neurosurg Psychiatry 1995; 58:393. 